\title{
Real-time observation of valence electron motion
}

\author{
Eleftherios Goulielmakis ${ }^{1 *}$, Zhi-Heng Loh ${ }^{2,3 *}$, Adrian Wirth ${ }^{1}$, Robin Santra ${ }^{4,5}$, Nina Rohringer ${ }^{6}$, \\ Vladislav S. Yakovlev ${ }^{1,7}$, Sergey Zherebtsov ${ }^{1}$, Thomas Pfeifer ${ }^{2,3} \dagger$, Abdallah M. Azzeer ${ }^{8}$, Matthias F. Kling ${ }^{1}$, \\ Stephen R. Leone ${ }^{2,3}$ \& Ferenc Krausz ${ }^{1,7}$
}

The superposition of quantum states drives motion on the atomic and subatomic scales, with the energy spacing of the states dictating the speed of the motion. In the case of electrons residing in the outer (valence) shells of atoms and molecules which are separated by electronvolt energies, this means that valence electron motion occurs on a subfemtosecond to few-femtosecond timescale $\left(1 \mathrm{fs}=10^{-15} \mathrm{~s}\right)$. In the absence of complete measurements, the motion can be characterized in terms of a complex quantity, the density matrix. Here we report an attosecond pump-probe measurement of the density matrix of valence electrons in atomic krypton ions ${ }^{1}$. We generate the ions with a controlled few-cycle laser field ${ }^{2}$ and then probe them through the spectrally resolved absorption of an attosecond extreme-ultraviolet pulse ${ }^{3}$, which allows us to observe in real time the subfemtosecond motion of valence electrons over a multifemtosecond time span. We are able to completely characterize the quantum mechanical electron motion and determine its degree of coherence in the specimen of the ensemble. Although the present study uses a simple, prototypical open system, attosecond transient absorption spectroscopy should be applicable to molecules and solid-state materials to reveal the elementary electron motions that control physical, chemical and biological properties and processes.

The millielectronvolt-scale spacing of vibrational energy levels implies that changes in molecular structure occur on a multifemtosecond timescale and can be accessed by femtosecond pump-probe spectroscopy ${ }^{4}$. Electronic phenomena in the valence band are one hundred to one thousand times faster and have remained elusive so far. Electronic coherence is the key to accessing them in real time. It has been studied in kinematically complete experiments ${ }^{5}$, from which the motion can be inferred but not directly observed. Apart from in the simplest systems, experimental techniques are unable to probe all degrees of freedom. In these cases, we have to deal with an open system, which can only be characterized in terms of ensembleaveraged quantities (observables) predicted by the system's density matrix. Under these circumstances, the synchrony of wave-packet dynamics in the specimens of the ensemble (that is, coherence) is indispensable and only time-resolved measurements can provide direct access to the observables of the motion. Combination of the powerful concepts of correlated measurement and high-harmonic spectroscopy $^{6-9}$ has recently uncovered signatures of electronic coherence and resultant dynamics in an ensemble of ionizing molecules within a temporal window of $\sim 1$ fs following ionization ${ }^{10}$. The degree and the persistence of coherence have not been measured and the method is limited to the scrutiny of systems with large $(\geq 10 \mathrm{eV})$ ionization potentials and of processes under strong-field influence.
Isolated attosecond extreme-ultraviolet (EUV) pulses ${ }^{3,11-13}$ lend themselves as a probe to overcome these limitations.

In this work, we introduce attosecond probe spectroscopy to study-as one of the simplest open quantum systems-krypton atoms ionized by a strong field. Strong-field ionization has been studied extensively ${ }^{14}$, without answering the question of whether it is able to create long-lived coherences. Insufficient temporal confinement of ionization, recollision and/or electron correlations under strong-field influence may affect electronic coherence in the emerging ions. Our study reveals that strong-field ionization by a waveformcontrolled $^{2}$, near-single-cycle ${ }^{13}$ laser pulse is capable of launching a broadband ( $\sim 0.7-\mathrm{eV}$ splitting) valence electron wave packet with a high degree of coherence $(g \approx 0.6)$ that persists without notable decay for much longer than $10 \mathrm{fs}$.

Attosecond probe spectroscopy can rely on the momentum or energy distribution of the liberated photoelectrons ${ }^{15}$ or transmitted photons as observables. Here we choose the latter option, transient absorption spectroscopy ${ }^{16-19}$, which can be extended to the scrutiny of condensed-matter phenomena whereas photoelectron spectroscopy is restricted to surfaces and gas-phase samples. Figure la illustrates our attosecond probing concept applied to the multiple ionization of krypton. Conventional spectroscopy ${ }^{20}$ shows that the ions are created in manifolds of states depicted by green boxes and denoted by $n l^{-i}$, indicating that, relative to the ground-state configuration of the atom, $i$ electrons are missing from the $n l$ subshell. An attosecond pulse carried at a photon energy of $\sim 80 \mathrm{eV}$ promotes the krypton ions created in their $4 p^{-i}$ manifolds to the $3 d^{-1} 4 p^{-(i-1)}$ core-hole states. The transitions lead to dips in the spectrum of the broadband EUV radiation transmitted through the ionized atomic ensemble (Fig. 1b), revealing populations and coherences on an attosecond-femtosecond timescale.

In our experiments, we ionized krypton atoms with sub-4-fs, waveform-controlled NIR laser pulses carried at a wavelength of $\sim 750 \mathrm{~nm}$ (ref. 13; wave period, $T_{\mathrm{L}} \approx 2.5 \mathrm{fs}$ ) and probed the emergence of ions and electronic dynamics within their $4 p^{-i}$ valence subshells with isolated sub-150-as EUV pulses carried at $\sim 80 \mathrm{eV}$ (Fig. 1c; for details, see Methods Summary). The target of the NIR pump/EUV probe exposure is a quasistatic cell filled with krypton atoms at densities on the order of $\sim 10^{18} \mathrm{~cm}^{-3}$. The EUV beam size at the focus is a small fraction of that of the NIR beam and, hence, ionization and accompanying electron dynamics are probed near the optical axis, where the radial dependence of the laser intensity can be neglected.

EUV absorption spectra are recorded as functions of the pumpprobe delay (negative delay means that the EUV probe precedes the NIR excitation) by a spectrometer downstream of the target. No

${ }^{1}$ Max-Planck-Institut für Quantenoptik, Hans-Kopfermann-Strasse 1, D-85748 Garching, Germany. ${ }^{2}$ Departments of Chemistry and Physics, University of California, Berkeley,

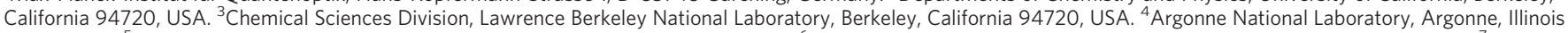

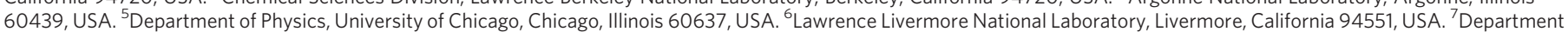

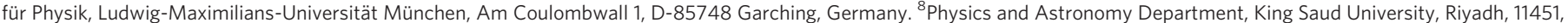
Kingdom of Saudi Arabia. †Present address: Max-Planck-Institut für Kernphysik, Saupfercheckweg 1, D-69117 Heidelberg, Germany.

*These authors contributed equally to this work. 
b

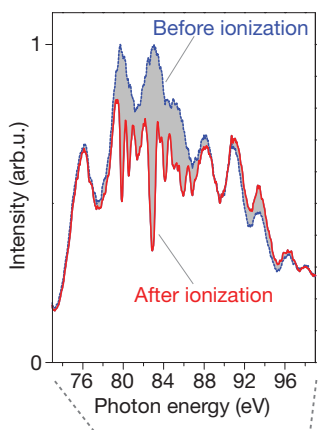

a 150

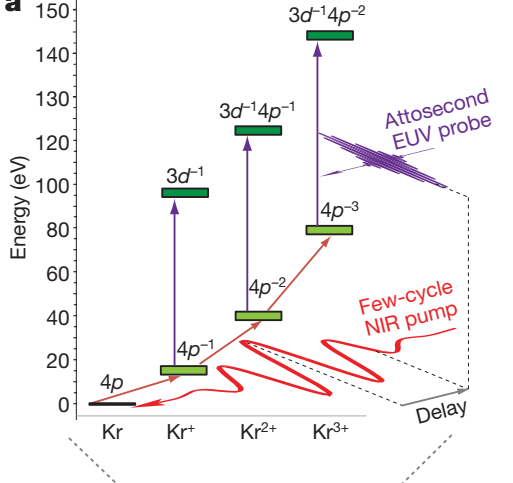

c

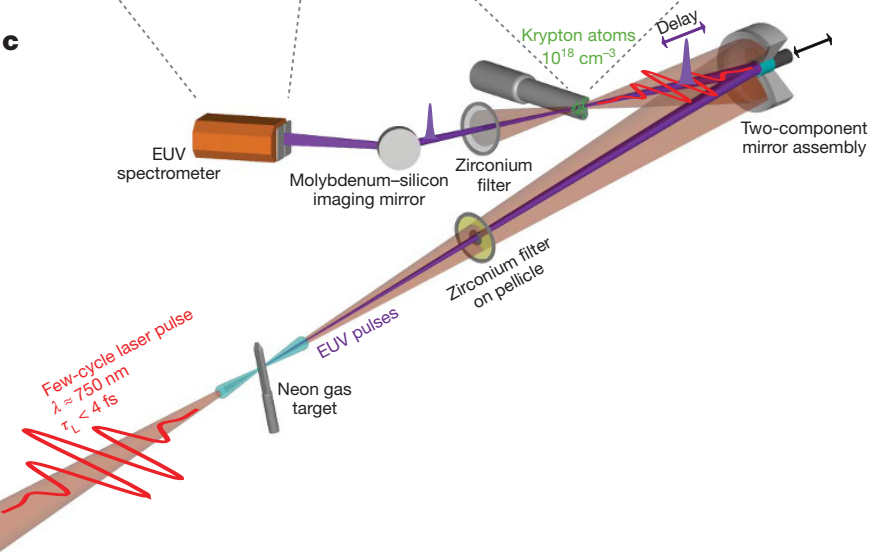

Figure $1 \mid$ Probing intra-atomic electron motion by attosecond absorption spectroscopy. a, The strong electric field of a near-infrared (NIR) laser pulse (in red) with a duration of $\tau_{\mathrm{L}}<4 \mathrm{fs}$ liberates electrons from the $4 p$ valence subshell of krypton atoms to generate singly charged $4 p^{-1}$, doubly charged $4 p^{-2}$ or triply charged $4 p^{-3}$ ions in the $4 p^{-1}, 4 p^{-2}$ and $4 p^{-3}$ manifolds of quantum states, respectively, by means of optical field ionization (indicated by red arrows). A subfemtosecond EUV pulse (in violet) with a carrier photon energy of $\sim 80 \mathrm{eV}$ is passed through the ions and promotes them to core-hole excited-state manifolds $3 d^{-1}, 3 d^{-1} 4 p^{-1}$ and $3 d^{-1} 4 p^{-2}$ (as indicated by the violet arrows). Transient EUV absorption spectra are acquired by recording the attosecond EUV pulse spectrum transmitted through the ionized gas target as a function of pump-probe delay with an EUV spectrometer. b, c, Spectral intensity distribution of the relevant part of the broadband attosecond probe pulse (b) and schematic of the experimental set-up (c). The pulse is transmitted through an ensemble of krypton atoms $\left(\sim 10^{18} \mathrm{~cm}^{-3}\right.$; interaction length, $\left.\sim 1 \mathrm{~mm}\right)$ before their ionization (blue curve) and after their ionization (red curve) by the laser pulse with a peak intensity of $\sim 7 \times 10^{14} \mathrm{~W} \mathrm{~cm}^{-2}$, as well as by several optical elements shown in $\mathrm{c}$ and discussed in detail in Methods. arb.u., arbitrary units.

high-order harmonic radiation emerging from the krypton target was detected in the spectral range of interest $(>60 \mathrm{eV})$. In Fig. 2, we plot a series of transient EUV absorption spectra for an on-axis peak laser intensity of $\sim 7 \times 10^{14} \mathrm{~W} \mathrm{~cm}^{-2}$, which is sufficiently high to produce singly, doubly and triply charged ions, as inferred from the appearance of absorption lines associated with electronic transitions in these ions. Our spectrometer has a resolution of $\sim 0.45 \mathrm{eV}$ and hence is unable to resolve the $\sim 88-\mathrm{meV}$ width $^{21}$ of the observed lines resulting from the $\sim 8$-fs core-hole decay of the $3 d^{-1} 4 p^{-(i-1)}$ states $^{22}$.

The appearance of different charge states delayed by approximately half the laser cycle with respect to each other is consistent with our understanding that the primary process in strong-field ionization is electron release near the field oscillation peaks by means of tunnelling or above-barrier escape. Attosecond transient absorption spectroscopy offers unprecedented insight into the dynamics of multiple ionization but theory must be further developed before this potential can be exploited. This is because the narrow natural linewidths of the absorption lines in the transmitted EUV light imply that

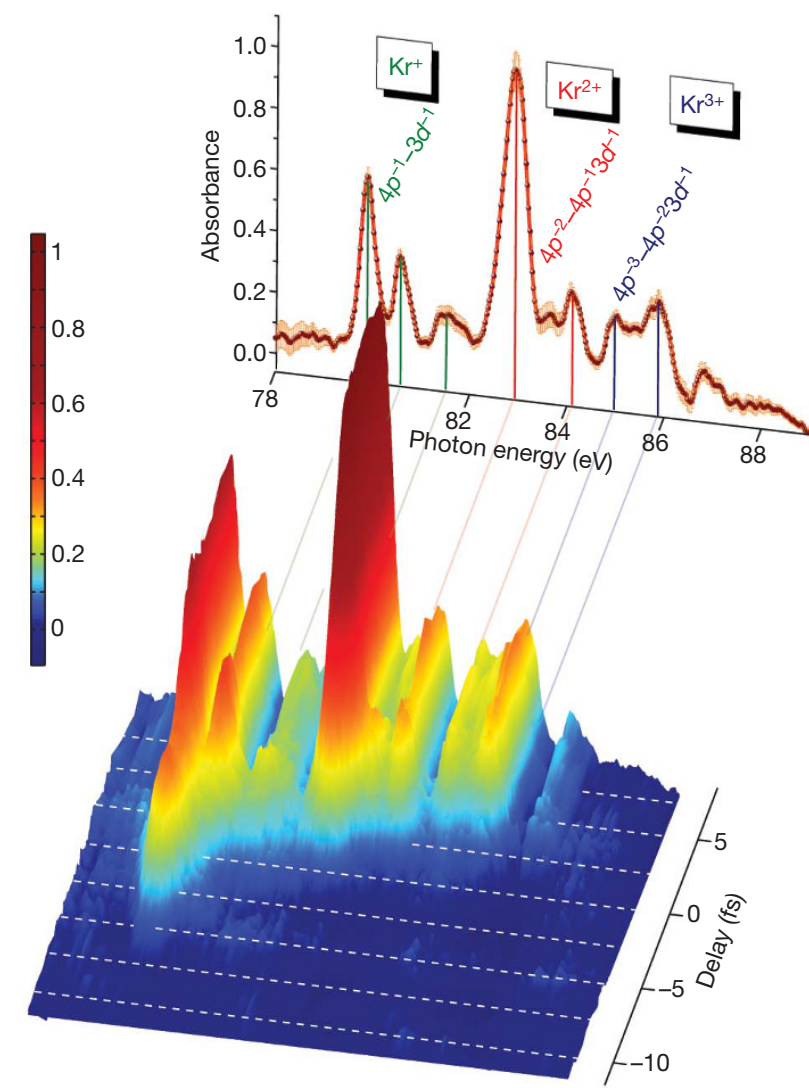

Figure 2 | Transient absorption spectra of krypton ions. Absorbance is defined as $A(E, \tau)=\ln \left(I_{\text {trans }}(E, \tau) / I_{0}(E)\right)$, where $I_{0}(E)$ is the spectral density recorded at a negative delay of $-10 \mathrm{fs}$, that is, the attosecond probe precedes the ionizing laser pulse by $10 \mathrm{fs}$ in the atomic sample, and $I_{\text {trans }}(E, \tau)$ is the spectral density recorded at a pump-probe delay $\tau$. The delay is varied in steps of 200 as. Error bars indicate the standard error of the mean values acquired from several spectra recorded at the same delay. The EUV probe pulse shows the formation of charge states up to $\mathrm{Kr}^{3+}$ as indicated in the spectrum shown in the background, which is recorded at $\tau \approx 10 \mathrm{fs}$. Disregarding a forerunner in the main $\mathrm{Kr}^{+}$line, the origin of which is unclear, the $\mathrm{Kr}^{2+}$ lines appear with a significant, well-resolved delay of about one-half the laser period $\left(T_{\mathrm{L}} /\right.$ $2 \approx 1.25 \mathrm{fs}$ ) after the $\mathrm{Kr}^{+}$lines, and the $\mathrm{Kr}^{3+}$ lines appear with approximately the same delay after the $\mathrm{Kr}^{2+}$ lines. The decrease in the neutral krypton population in the atomic sample manifests itself as a reduction of the absorption in the range $91-93 \mathrm{eV}$, where neutral krypton atoms absorb resonantly. Relative occupations between the ionic states $\mathrm{Kr}^{+}, \mathrm{Kr}^{2+}$ and $\mathrm{Kr}^{3+}$ are estimated as $N_{K r^{+}}: N_{K r^{2+}}: N_{K r^{3+}}=1: 0.875: 0.25$.

they result from a coherent polarization response of the atomic ensemble that extends over several femtoseconds after the EUV pulse hits the sample. During this time, the strong laser field is still present and may affect the atomic polarization response. Modelling of this interaction is beyond the scope of this work; here we focus on dynamics occurring in $\mathrm{Kr}^{+}$ions after the ionizing field has vanished, where this complication does not arise.

It is known from photoelectron ${ }^{23,24}$ and photoabsorption ${ }^{18,25}$ spec- $^{-}$ troscopy that strong-field ionization populates not only the ground state but also excited electronic states of the emerging ions. Exposure of krypton atoms to a strong, low-frequency laser field is expected to generate $\mathrm{Kr}^{+}$ions predominantly in their $4 p_{j=3 / 2}^{-1}$ ground-state manifold and the $4 p_{j=1 / 2}^{-1}$ excited-state manifold, comprising four $\left(m_{j=3 / 2}=-3 / 2,-1 / 2,1 / 2,3 / 2\right)$ and two $\left(m_{j=1 / 2}=-1 / 2,1 / 2\right)$ states, respectively ( $j$ denotes the total angular momentum and $m_{j}$ denotes its projection on the $z$ axis). The fine-structure (or spin-orbit) energy splitting between the two manifolds is $\Delta E_{\mathrm{SO}}=0.67 \mathrm{eV}$ (ref. 26). However, the critical question is whether they can be populated coherently during strong-field ionization so as to allow the creation of subsequent wave-packet motion in the valence shell ${ }^{27}$. 
We have numerically modelled the interaction of krypton atoms with a strong laser field, as described in ref. 1 . The populations of the six relevant quantum states of $\mathrm{Kr}^{+}$and the coherence between these states are respectively given by the diagonal elements $\rho_{j, j^{\prime}=j}^{\left(m_{j}\right)}$ and the corresponding off-diagonal elements of the reduced density matrix of the atomic ensemble (Supplementary Information, section I). Solving the time-dependent Schrödinger equation for a few-cycle ionizing NIR field, linearly polarized along the quantization axis, yields the evolution of the density matrix elements as depicted in Fig. 3. By the end of the laser pulse ( $t \geq 3 \mathrm{fs}$ ), $\sim 30 \%$ of the atoms are ionized, with a hole emerging in the $\left(4 p_{j}, m_{j}\right)$ or $\left(4 p_{j},-m_{j}\right)$ orbital with a relative population of $\rho_{3 / 2,3 / 2}^{(1 / 2)}+\rho_{3 / 2,3 / 2}^{(-1 / 2)}=2 \rho_{3 / 2,3 / 2}^{(1 / 2)}=$ $0.69, \rho_{1 / 2,1 / 2}^{(1 / 2)}+\rho_{1 / 2,1 / 2}^{(-1 / 2)}=2 \rho_{1 / 2,1 / 2}^{(1 / 2)}=0.26$ and $\rho_{3 / 2,3 / 2}^{(3 / 2)}+\rho_{3 / 2,3 / 2}^{(-3 / 2)}=$ $2 \rho_{3 / 2,3 / 2}^{(3 / 2)}=0.05$ (Fig. 3a). The hole populations increase in subfemtosecond steps near the oscillation peaks of the ionizing laser field depicted by the dashed line. Only states with the same value of $m_{j}$ can form a coherent superposition state characterized by a non-zero value of the corresponding off-diagonal element of the density matrix. In our case, this condition is fulfilled by the $\left(4 p_{3 / 2}^{-1}\right.$, $\left.m_{j}= \pm 1 / 2\right)$ and $\left(4 p_{1 / 2}^{-1}, m_{j}= \pm 1 / 2\right)$ states $^{1}$. The degree of coherence between these states can be characterized by the parameter

$$
g(t)=\frac{\left|\rho_{3 / 2,1 / 2}^{(1 / 2)}\right|}{\sqrt{\rho_{3 / 2,3 / 2}^{(1 / 2)} \rho_{1 / 2,1 / 2}^{(1 / 2)}}}
$$

Perfect coherence corresponds to $g=1$, whereas incoherent superpositions yield $g=0$. In Fig. $3 \mathrm{~b}$, we plot the half-cycle-averaged $g(t)$ for pulse durations of $3.8 \mathrm{fs}$ and $7.6 \mathrm{fs}$ (full-width at half-maximum of the intensity envelope).
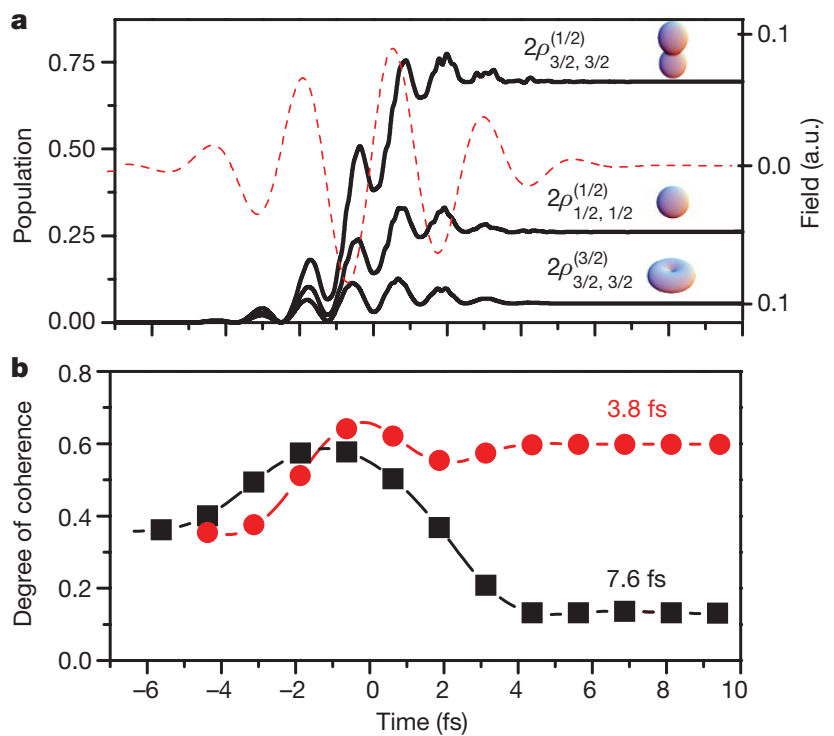

Figure 3 | Build-up of electronic coherence in $\mathrm{Kr}^{+}$produced by optical field ionization (theory). a, Temporal evolution of the diagonal elements of the reduced density matrix of $\mathrm{Kr}^{+}$in the presence of a $750-\mathrm{nm}, 3.8$-fs laser pulse with a peak intensity of $\sim 3 \times 10^{14} \mathrm{~W} \mathrm{~cm}^{-2}$ and a sinusoidal waveform. The notation of the matrix elements is explained in the text. The populations have been normalized such that the trace of the reduced density matrix at $\tau=10 \mathrm{fs}$ equals one. The dashed line shows the electric field of the laser pulse (in atomic units (a.u.)) used in the simulations. The hole density distributions of the corresponding orbitals of the $4 p$ subshell are also depicted. $\mathbf{b}$, The degree of coherence, $g(t)$ (see equation (1)), averaged over time intervals of the laser half-cycle $(\sim 1.25 \mathrm{fs})$, is shown with red dots. Squares show the half-cycle-averaged degree of coherence for an ionizing laser pulse with a duration of $7.6 \mathrm{fs}$ at the same peak intensity. The degree of coherence is defined such that, after the NIR pump pulse, it equals one for a perfectly coherent hole wave packet. The detailed behaviour of the degree of coherence during ion formation is not yet understood.
The evolution of coherence shown in Fig. 3b can be understood intuitively. At every intense wave crest, a fraction of the atoms is ionized and a hole spin-orbit wave packet is launched in the $4 p$ valence subshell by populating the $4 p^{-1}$ manifold. Spin-orbit wave packets launched in ions that are generated at a given wave crest have a fairly well-defined phase with respect to the wave crest and evolve with a half-period of $3.1 \mathrm{fs}$. As a consequence, their phase has changed by $0.4 \pi$ by the time the next wave crest arrives (after one half of the laser period, $T_{\mathrm{L}} / 2=1.25 \mathrm{fs}$ ), such that ions produced at this wave crest are phase-shifted with respect to the ions generated previously. In the 3.8-fs pulse, ionization is confined to two to three wave crests, that is, to within a single laser period. Therefore, the final population of the $4 p^{-1}$ manifold builds up within less than the 3.1-fs half-period, ensuring that the ions produced at the individual wave crests are substantially in phase. This gives rise to a final ion ensemble with a high degree of coherence $(g \approx 0.6)$. By contrast, in the 7.6 -fs pulse, the build-up of $4 p^{-1}$ population takes longer, leading to a wider distribution of relative phases among the ions created and, hence, to a strongly reduced degree of coherence of the ion ensemble $(g \approx 0.13)$. Our simulations reveal that, in the absence of a tunable excitation period, the creation of a persisting electronic coherence critically relies on the confinement of excitation to a time interval that is comparable to the characteristic timescale of the resultant wavepacket motion. These simulations also reveal that field ionization by multicycle pulses ${ }^{10}$ generally creates incoherent ion ensembles.

When the coherent broadband EUV probe promotes the system from the components of its superposition state into a common final state, quantum interference leads to a temporal modulation of the transition probability: its depth is indicative of the degree of coherence of the probed superposition state, and its variation with pumpprobe delay reveals the temporal evolution of the system. In our case, strong-field ionization is predicted to create a hole superposition in the $4 p_{j=3 / 2}^{-1}$ ground-state manifold and the $4 p_{j=1 / 2}^{-1}$ excited-state manifold of $\mathrm{Kr}^{+}$ions (Fig. 4a). A broadband pulse carried at $\sim 80 \mathrm{eV}$ promotes the ions from these states-by means of dipoleallowed transitions - into the $3 d_{3 / 2}^{-1}$ manifold by creating a core-level vacancy. A transition from the $4 p_{3 / 2}^{-1}$ states to the $3 d_{5 / 2}^{-1}$ states is also possible, but the $4 p_{1 / 2}^{-1} \rightarrow 3 d_{5 / 2}^{-1}$ transition is forbidden by electric dipole selection rules. Figure $4 \mathrm{~b}$ shows the simulated absorption cross-section of $\mathrm{Kr}^{+}$for the above transitions (see Supplementary Information, section I, for details) as a function of pump-probe delay, for the simulation parameters given in Fig. 3.

The high degree of coherence between the $4 p_{1 / 2}^{-1}$ and $4 p_{3 / 2}^{-1}$ states (Fig. 3b) is predicted to translate into modulation of the absorption cross-sections as functions of pump-probe delay for the $4 p_{3 / 2}^{-1} \rightarrow 3 d_{3 / 2}^{-1}$ and $4 p_{1 / 2}^{-1} \rightarrow 3 d_{3 / 2}^{-1}$ transitions (Fig. $4 \mathrm{~b}$ ), owing to a temporal variation of the relative phase, $\phi$, between the $4 p_{1 / 2}^{-1}$ and $4 p_{3 / 2}^{-1}$ states

$$
\begin{aligned}
\phi(t) & =\frac{E_{|4 p, j=1 / 2\rangle}-E_{|4 p, j=3 / 2\rangle}}{\hbar} t+\phi_{0} \\
& \equiv \frac{\Delta E_{\mathrm{SO}}}{\hbar} t+\phi_{0} \equiv \frac{2 \pi}{T_{\mathrm{SO}}} t+\phi_{0}
\end{aligned}
$$

where $E_{|4 p, j=1 / 2\rangle}$ is the energy of the $4 p_{1 / 2}^{-1}$ state, $E_{|4 p, j=3 / 2\rangle}$ is the energy of the $4 p_{3 / 2}^{-1}$ state and $\hbar$ is Planck's constant divided by $2 \pi$. Here $\Delta E_{\mathrm{SO}}=0.67 \mathrm{eV}$ implies a wave-packet oscillation period of $T_{\mathrm{SO}}=6.2 \mathrm{fs}$. The modulation is most pronounced in the weaker $4 p_{3 / 2}^{-1} \rightarrow 3 d_{3 / 2}^{-1}$ transition and is absent from the $4 p_{3 / 2}^{-1} \rightarrow 3 d_{5 / 2}^{-1}$ transition, which is insensitive to the quantum superposition. In equation (2), $\phi(t)$ describes the relative phase between the two states after the NIR pulse. For a given NIR waveform, $\phi_{0}$ is a nontrivial, well-defined quantity, which will become experimentally measurable by combining attosecond absorption spectroscopy with attosecond streaking to determine the NIR waveform and its timing with respect to the attosecond probe pulse with attosecond accuracy. Access to the phase during the laser pulse has been possible for short-lived coherences ${ }^{10}$. The experimental data (Fig. 4c) clearly show the predicted consequences of the 

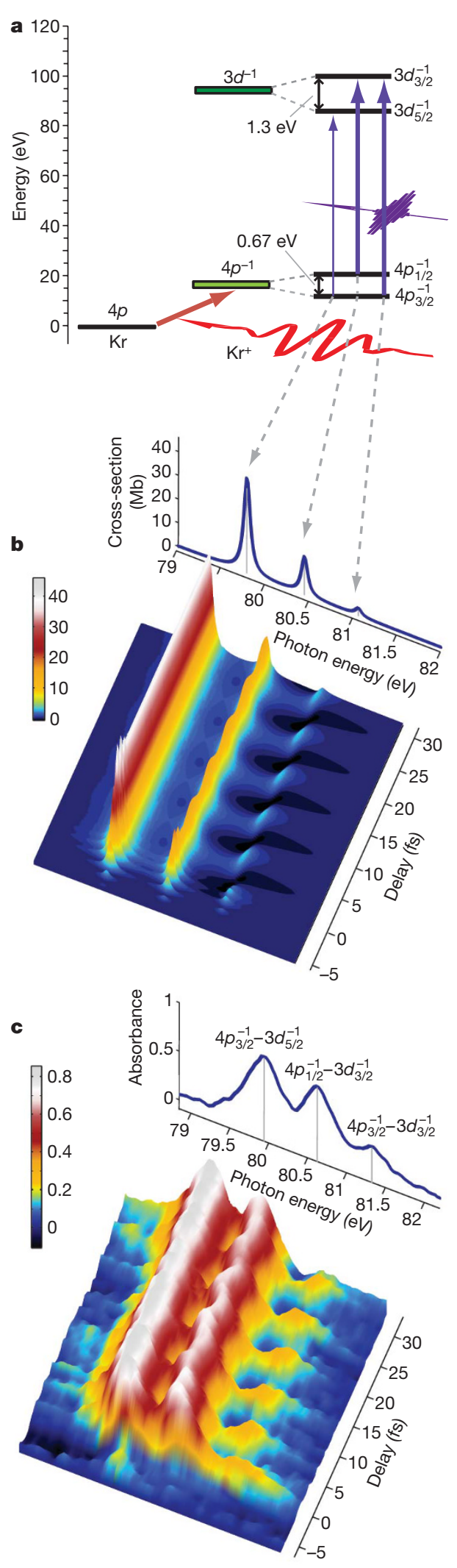

wave-packet motion described by equation (2), including the deep amplitude modulation of the $4 p_{3 / 2}^{-1} \rightarrow 3 d_{3 / 2}^{-1}$ absorption line as well as the energy modulation of the lines, with the latter resulting from absorptive and dispersive terms in the transient absorption cross$\operatorname{section}^{16}$ (see equation (1) in Supplementary Information).

Components of the reduced density matrix of the system are determined by adjusting them along with $T_{\mathrm{SO}}$ such that the model's
Figure 4 | Attosecond absorption spectroscopy reveals intra-atomic electron wave-packet motion in $\mathrm{Kr}^{+}$. a, Energy-level diagram showing the spin-orbit splitting of the $4 p$ and $3 d$ subshells in $\mathrm{Kr}^{+}$. A sub-4-fs NIR laser pulse (red wave) liberates an electron from the $4 p$ subshell and leaves the ensemble of ions in a coherent superposition of $4 p_{3 / 2}^{-1}$ and $4 p_{1 / 2}^{-1}$ states. Single-photon EUV absorption promotes the ions from these states to the core-excited $3 d_{3 / 2}^{-1}$ state. b, Simulated transient EUV absorption spectra reveal characteristic modulations present in the $4 p_{3 / 2}^{-1} \rightarrow 3 d_{3 / 2}^{-1}$ and $4 p_{1 / 2}^{-1} \rightarrow 3 d_{3 / 2}^{-1}$ transitions as functions of pump-probe delay. The modulation depth is highly sensitive to the degree of coherence, $g(t)$. Mb, megabarn. c, False-colour plot of an attosecond absorption spectrogram comprising 40 transient absorption spectra recorded at delays increased in steps of $1 \mathrm{fs}$ with a sub-4-fs, $\sim 750$-nm laser pump and a sub-150-as, $\sim 80-\mathrm{eV}$ EUV probe. The reference spectrum was recorded at $-6 \mathrm{fs}$. The absorption spectrum plotted in the background is taken at a delay of $30 \mathrm{fs}$. The linewidths are determined by the $\sim 0.45-\mathrm{eV}$ resolution of our EUV spectrometer. The lower modulation depth of the $4 p_{1 / 2}^{-1} \rightarrow 3 d_{3 / 2}^{-1}$ transition relative to the calculations shown in $\mathbf{b}$ is a result of the spectral resolution being limited in comparison with the $\sim 88-\mathrm{meV}$ natural linewidth of the studied transitions. The zero of the delay scale is set to coincide with the instant when the main $4 p_{3 / 2}^{-1} \rightarrow 3 d_{5 / 2}^{-1}$ absorption line reaches $95 \%$ of its quasistationary value.

prediction agrees best with spectra recorded over the photon energy range $78.5-82 \mathrm{eV}$ and the pump-probe delay range 3-33 fs (Supplementary Information, section III). This procedure has yielded (for $t \geq 3$ fs) $\rho_{3 / 2,3 / 2}^{(1 / 2)}+\rho_{3 / 2,3 / 2}^{(-1 / 2)}=0.42 \pm 0.10, \quad \rho_{1 / 2,1 / 2}^{(1 / 2)}+\rho_{1 / 2,1 / 2}^{(-1 / 2)}=$ $0.35 \pm 0.03, \quad \rho_{3 / 2,3 / 2}^{(3 / 2)}+\rho_{3 / 2,3 / 2}^{(-3 / 2)}=0.23 \pm 0.08, \quad g=0.63 \pm 0.17$ and $T_{\mathrm{SO}}=6.3 \pm 0.1 \mathrm{fs}$. This value for $T_{\mathrm{SO}}$ is in excellent agreement with the one derived from $\Delta E_{\mathrm{SO}}$ (ref. 26). The degree of coherence agrees well with the predictions in Fig. 3, verifying that few-cycle ionization is capable of creating robust electronic coherence. It survives over a timescale of multiple tens of femtoseconds, allowing electron wave packets to affect structural dynamics once launched in molecular orbitals.

Fig. 5a displays the mean optical density of the $4 p_{3 / 2}^{-1} \rightarrow 3 d_{3 / 2}^{-1}$ absorption line as a function of pump-probe delay over the energy range $81.20-81.45 \mathrm{eV}$ (black dots) in comparison with the prediction of our model with the parameters given above (red line). To reconstruct the quantum mechanical motion of the electron wave packet, the temporal evolution of the quantum phase $\phi(t)$ defined by equation (2) must also be retrieved. Attosecond absorption spectroscopy allows this retrieval over a multifemtosecond time interval with a resolution matched to the electronic timescale (Fig. 5b). The uncertainty in the obtained values of the quantum phase (of $\sim \pi / 5$ ) translates-using equation (2)-into a temporal resolution of $\sim 0.6$ fs. In Fig. 5 b, we plot the evolution of the quantum phase versus the time elapsed since the main absorption line of $\mathrm{Kr}^{+}$reached 95\% of its stationary value. In this frame of reference, the electron (or hole) density distribution can be reconstructed at any instant after the ionizing laser pulse with subfemtosecond accuracy. We plot the result of this reconstruction at a few selected instants separated by $1 \mathrm{fs}$ (Supplementary Information, section II). Measuring $\phi_{0}$ along with the amplitude of the off-diagonal matrix element as a function of the laser waveform, which can be determined using attosecond streaking $^{11}$, will provide insight into the dynamics of optical field ionization and the concomitant formation of intra-atomic electron wave packets. Through comparisons with model predictions, this measured phase will provide a new, sensitive test of the modelling of strongfield ionization of multi-electron atoms, a process that is far from being well understood.

Attosecond transient absorption spectroscopy extends real-time insight into microscopic motion, from nuclear wave-packet ${ }^{4}$ and Rydberg wave-packet ${ }^{28}$ dynamics to electrons in the valence shell. Its unique features, that is, gentle probing owing to the absence of strong fields, the capacity to study processes within condensed matter, and wide applicability to materials with arbitrary ionization potential, render this approach ideal for attosecond real-time observation of electronic and concomitant processes in atoms, molecules and solids ${ }^{29,30}$. 


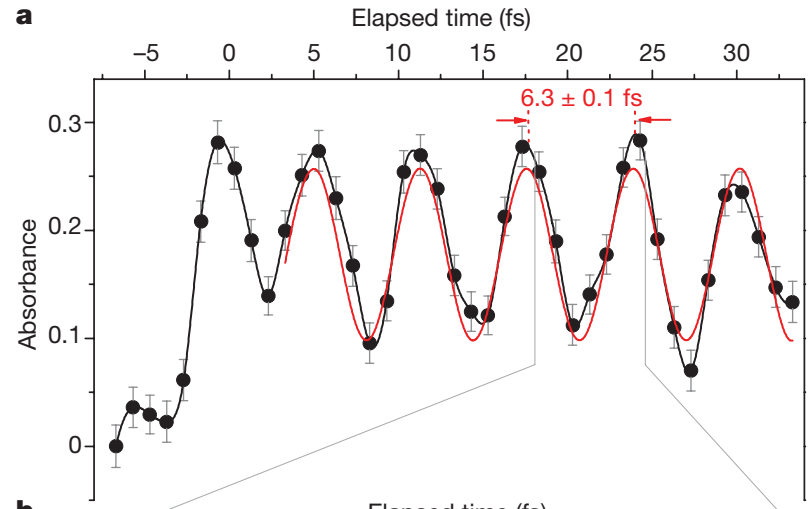

b

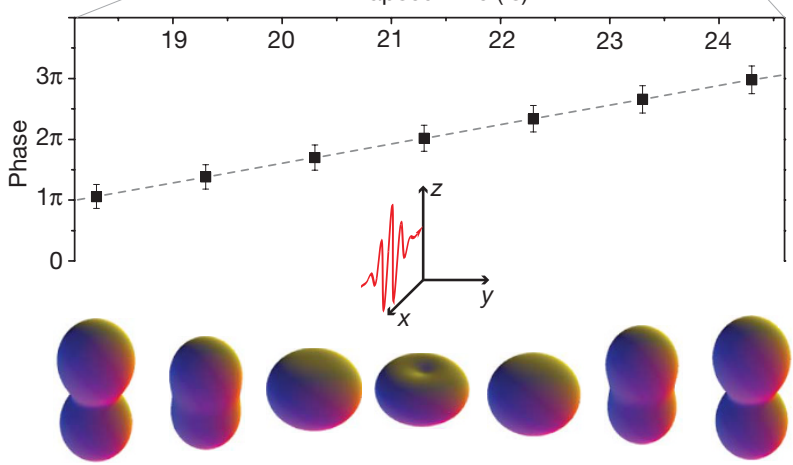

Figure 5 | Reconstruction of valence-shell electron wave-packet motion. a, Absorbance (dots) averaged over the photon energy range $81.20-81.45 \mathrm{eV}$ corresponding to the $4 p_{3 / 2}^{-1} \rightarrow 3 d_{3 / 2}^{-1}$ transition (see Fig. $4 \mathrm{c}$ ), as a function of time elapsed since zero as defined in the text. The full line shows the result of our modelling for the values of the fit parameters given in the text. The modulation occurs with a period of $6.3 \pm 0.1 \mathrm{fs}$. Error bars depict the standard error of the values extracted from several data sets recorded under identical experimental conditions. $\mathbf{b}$, Quantum phase of the $4 p$ superposition state $\phi(t)$ (see equation (2)), as retrieved from the measured attosecond absorption spectrogram shown in Fig. 4c. Uncertainty in the values, resulting from our measurement and modelling, indicates accuracy of reconstruction of the superposition of $\sim \pi / 5$. The lower diagram shows ensemble-averaged hole density distributions in the $4 p$ subshell of $\mathrm{Kr}^{+}$reconstructed from the measured $\phi(t)$ and the measured components of the density matrix, at instants separated by $1 \mathrm{fs}$, within an interval of $17-25 \mathrm{fs}$ following ionization.

\section{METHODS SUMMARY}

We sent sub-4-fs, $0.3-\mathrm{mJ}$ NIR laser pulses at a wavelength of $\sim 750 \mathrm{~nm}$ into a neon-filled tube to generate EUV pulses by means of high-harmonic generation. The collinear NIR and EUV beams were then passed through a filter assembly and focused in a quasistatic gas cell containing the krypton gas at a pressure of about 80 mbar. The EUV pulse hitting the target was delayed with respect to the NIR laser pulse and had a duration of less than 150 as. A broadband molybdenum-silicon multilayer mirror imaged, in one transverse dimension, the attosecond EUV beam transmitted through the krypton gas target to the entrance slit of an EUV spectrometer used for measuring the spectral intensity distribution of the transmitted beam.

Full Methods and any associated references are available in the online version of the paper at www.nature.com/nature.

\section{Received 25 September 2009; accepted 24 May 2010.}

1. Rohringer, N. \& Santra, R. Multichannel coherence in strong-field ionization. Phys. Rev. A 79, 053402 (2009).

2. Baltuska, A. et al. Attosecond control of electronic processes by intense light fields. Nature 421, 611-615 (2003).

3. Hentschel, M. et al. Attosecond metrology. Nature 414, 509-513 (2001).

4. Zewail, A. H. Femtochemistry: atomic-scale dynamics of the chemical bond. J. Phys. Chem. A 104, 5660-5694 (2000).

5. Schöffler, M. S. et al. Ultrafast probing of core hole localization in $\mathrm{N}_{2}$. Science 320, 920-923 (2008)
6. Niikura, H. et al. Sub-laser-cycle electron pulses for probing molecular dynamics. Nature 417, 917-922 (2002)

7. Niikura, H. et al. Probing molecular dynamics with attosecond resolution using correlated wave packet pairs. Nature 421, 826-829 (2002).

8. Niikura, H., Villeneuve, D. M. \& Corkum, P. B. Mapping attosecond electron wave packet motion. Phys. Rev. Lett. 94, 083003 (2005).

9. Baker, S. et al. Probing proton dynamics in molecules on an attosecond time scale. Science 312, 424-427 (2006).

10. Smirnova, O. et al. High harmonic interferometry of multi-electron dynamics in molecules. Nature 460, 972-977 (2009).

11. Kienberger, R. et al. Atomic transient recorder. Nature 427, 817-821 (2004).

12. Sansone, G. et al. Isolated single-cycle attosecond pulses. Science 314, 443-446 (2006).

13. Goulielmakis, E. et al. Single-cycle nonlinear optics. Science 320, 1614-1617 (2008).

14. Brabec, T. Strong Field Laser Physics (Springer, 2008).

15. Yudin, G. L. et al. Attosecond photoionization of coherently coupled electronic states. Phys. Rev. A 72, 051401 (2005)

16. Pollard, W. T., Lee, S.-Y. \& Mathies, R. A. Wave packet theory of dynamic absorption spectra in femtosecond pump-probe experiments. J. Chem. Phys. 92, 4012-4029 (1990)

17. Mathies, R. A. et al. Direct observation of the femtosecond excited-state cis-trans isomerization in bacteriorhodopsin. Science 240, 777-779 (1988)

18. Loh, Z.-H. et al. Quantum state-resolved probing of strong-field-ionized xenon atoms using femtosecond high-order harmonic transient absorption spectroscopy. Phys. Rev. Lett. 98, 143601 (2007).

19. Loh, Z.-H. \& Leone, S. R. Ultrafast strong-field dissociative ionization dynamics of $\mathrm{CH}_{2} \mathrm{Br}_{2}$ probed by femtosecond soft $\mathrm{X}$-ray transient absorption spectroscopy. J. Chem. Phys. 128, 204302 (2008).

20. Southworth, S. H. et al. K-edge X-ray-absorption spectroscopy of laser-generated $\mathrm{Kr}^{+}$and $\mathrm{Kr}^{2+}$. Phys. Rev. A 76, 043421 (2007).

21. Jurvansuu, M., Kivimäki, A. \& Aksela, S. Inherent lifetime widths of $\operatorname{Ar} 2 p^{-1}, \mathrm{Kr}$ $3 d^{-1}, \mathrm{Xe} 3 d^{-1}$, and Xe $4 d^{-1}$ states. Phys. Rev. A 64, 012502 (2001).

22. Drescher, M. et al. Time-resolved atomic inner-shell spectroscopy. Nature 419, 803-807 (2002).

23. Rottke, H., Ludwig, J. \& Sandner, W. 'Short' pulse MPI of xenon: the ${ }^{2} P_{1 / 2}$ ionization channel. J. Phys. B 29, 1479 (1996).

24. Gubbini, E. et al. Core relaxation in atomic ultrastrong laser field ionization. Phys. Rev. Lett. 94, 053602 (2005).

25. Young, L. et al. X-ray microprobe of orbital alignment in strong-field ionized atoms. Phys. Rev. Lett. 97, 083601 (2006).

26. Saloman, E. B. Energy levels and observed spectral lines of krypton, $\mathrm{Kr} /$ through $\mathrm{Kr}$ XXXVI. J. Phys. Chem. Ref. Data 36, 215-386 (2007)

27. Santra, R., Dunford, R. W. \& Young, L. Spin-orbit effect on strong-field ionization of krypton. Phys. Rev. A 74, 043403 (2006).

28. Jones, R. R. \& Noordam, L. D. Electronic wavepackets. Adv. At. Mol. Opt. Phys. 38, 1-38 (1998)

29. Bucksbaum, P. H. The future of attosecond spectroscopy. Science 317, 766-769 (2007).

30. Krausz, F. \& Ivanov, M. Y. Attosecond physics. Rev. Mod. Phys. 81, 163-234 (2009).

Supplementary Information is linked to the online version of the paper at www.nature.com/nature.

Acknowledgements We thank U. Kleineberg, M. Hofstetter and M. Fiess for invaluable contributions. This work was supported by the Max Planck Society, the Nobel Program of King Saud University and the DFG Cluster of Excellence: Munich Centre for Advanced Photonics (http://www.munich-photonics.de). E.G. acknowledges a Marie-Curie Reintegration grant (MERG-CT-2007-208643). A.W., S.Z. and M.F.K. acknowledge support by the Emmy Noether programme of the DFG. Z.-H.L., T.P. and S.R.L. acknowledge support from the Air Force Office of Scientific Research (FA9550-04-1-0242), the National Science Foundation (CHE-0742662 and EEC-0310717) and the Director, Office of Science, Office of Basic Energy Sciences, US Department of Energy (DE-AC02-05-CH11231). T.P. acknowledges support from the MPRG program of the MPG. R.S. is supported by the Office of Basic Energy Sciences, Office of Science, US Department of Energy (DE-AC02-06CH11357). Part of this work was performed under the auspices of the US Department of Energy by Lawrence Livermore National Laboratory (DE-AC52-07NA27344). S.R.L. gratefully acknowledges appointment as a Miller Research Professor in the Miller Institute for Basic Research in Science.

Author Contributions E.G., Z.-H.L. and A.W. conceived and designed the experiments; E.G., A.W. and Z.-H.L. performed the measurements; A.W., Z.-H.L., E.G., T.P., S.Z., A.M.A., M.F.K., S.R.L and F.K. evaluated, analysed and interpreted the experimental data; and R.S., N.R. and V.S.Y. performed the theoretical modelling. All authors discussed the results and contributed to the final manuscript.

Author Information Reprints and permissions information is available at www.nature.com/reprints. The authors declare no competing financial interests. Readers are welcome to comment on the online version of this article at www.nature.com/nature. Correspondence and requests for materials should be addressed to E.G. (elgo@mpq.mpg.de), S.R.L. (srl@berkeley.edu) or F.K. (krausz@lmu.de). 


\section{METHODS}

Carrier-envelope-phase-controlled, sub-4-fs, 0.3-mJ NIR laser pulses carried at a wavelength of $\sim 750 \mathrm{~nm}$ and delivered at a repetition rate of $3 \mathrm{kHz}$ are focused into a neon-filled tube to generate EUV pulses by means of high-harmonic generation (Fig. 1c). On their way towards a two-component, concentric mirror module, the collinear NIR and EUV beams pass through a filter assembly consisting of a 150-nmthick zirconium foil and an ultrathin pellicle. The small-divergence EUV beam is transmitted through the zirconium filter covering a circular spot $\sim 3 \mathrm{~mm}$ in diameter, whereas the NIR beam is efficiently blocked by this filter. The outer part of the (more divergent) NIR beam is transmitted by the pellicle carrying the zirconium foil.

The EUV beam transmitted through the circular zirconium filter has a diameter of $\sim 3 \mathrm{~mm}$ when hitting the internal part of the double mirror assembly, which also has a diameter of $\sim 3 \mathrm{~mm}$. This inner mirror is covered with a molybdenum-silicon multilayer with a reflectance of $\sim 2.5 \%$ over a $28-\mathrm{eV}$ (full-width at half-maximum) band centred at $\sim 80 \mathrm{eV}$. In these experiments, spectral filtering and the intensity of the driving field yield pulses with $\sim 15-\mathrm{eV}$ bandwidth. The NIR beam is reflected by the outer part of the mirror assembly, which is coated with silver. Both mirrors are deposited on a super-polished substrate with a radius of curvature of $25 \mathrm{~cm}$. Both beams are focused in a quasistatic gas cell formed by a nickel tube, containing the krypton gas at a pressure of about 80 mbar. The effective interaction length in the krypton gas target is $\sim 1 \mathrm{~mm}$. The EUV pulses hitting the target have a duration of less than 150 as as verified by attosecond streak-camera measurements. It is delayed with respect to the laser pulse by the focusing molybdenum-silicon mirror mounted on a piezo-controlled translation stage. A motorized aperture installed downstream of the source adjusts the on-axis NIR intensity on target (not shown) between zero and $\sim 7 \times 10^{14} \mathrm{~W} \mathrm{~cm}^{-2}$. A second zirconium foil, installed behind the krypton gas cell, prevents the NIR light from entering the EUV spectrometer. A broadband molybdenum-silicon multilayer mirror images, in one transverse dimension, the transmitted EUV beam from the krypton gas target to the entrance slit of an EUV spectrometer used for measuring the spectral intensity distribution of the attosecond EUV beam transmitted through the ionized target. 